\title{
Effect of ranibizumab on levels of IL-6 and VEGF in peripheral blood and aqueous humor of glaucoma rat model and association of IL-6 and VEGF with optic nerve damage
}

\author{
YANLING SONG* ${ }^{*}$ QIUJIE SONG* ${ }^{*}$ LING LI, JINFENG XU and XINGQIANG LIU \\ Department of Ophthalmology, People's Hospital of Dongying, Dongying, Shandong 257091, P.R. China
}

Received December 26, 2017; Accepted June 19, 2018

DOI: $10.3892 /$ etm.2018.6441

\begin{abstract}
This study aims to investigate the effect of intravitreal injection of ranibizumab on glaucoma rat model and its effect on optic nerve injury. A total of 125 SD rats were used to establish glaucoma models. Of them, 80 well-established model were selected, 40 of which received intravitreal injections of ranibizumab and were assigned to the observation group. The remaining 40 received no drug and were assigned to the control group. After rats were sacrificed at the timepoints, the retina was harvested. Numbers of retinal ganglion cells (RGCs) were counted under a fluorescence microscope. In the meantime, levels of interleukin-6 (IL-6) and vascular endothelial growth factor (VEGF) in peripheral blood and aqueous humor were determined. The levels of IL-6 and VEGF in peripheral blood and aqueous humor decreased gradually over the time of treatment in the observation group $(\mathrm{P}<0.05)$. In the control group the levels of IL-6 in peripheral blood and aqueous humor increased gradually over time $(\mathrm{P}<0.05)$. At the same time-point, the IL-6 level was higher in the control group than that in the observation group $(\mathrm{P}<0.05)$. The VEGF level in the observation group was lower in day 21 than that in day $7(\mathrm{P}<0.05)$. The VEGF level was higher in the control group than that in the observation group $(\mathrm{P}<0.05)$. The number of RGCs in the observation group increased gradually over the time of treatment, and there were significant differences in the number of RGCs between day 7 and 14 , as well as day 14 and $21(\mathrm{P}<0.05)$. The number of RGCs in the control group decreased gradually over time $(\mathrm{P}<0.05)$. The levels of IL- 6 and VEGF were all negatively correlated with the number of RGCs, and the correlation coefficient $r$
\end{abstract}

Correspondence to: Dr Qiujie Song, Department of Ophthalmology, People's Hospital of Dongying, 317 Dongchengnanyi Road, Dongying, Shandong 257091, P.R. China

E-mail: songqiujie12@163.com

*Contributed equally

Key words: ranibizumab, glaucoma, interleukin-6, vascular endothelial growth factor optic nerve injury, retinal ganglion cell and P-value were -0.743 and 0.012 , and -0.675 and 0.022 , respectively. Ranibizumab attenuated optic nerve injury by reducing levels of IL- 6 and VEGF in peripheral blood and aqueous humor of glaucoma rat model.

\section{Introduction}

Elevated intraocular pressure, intermittent or persistent, is often a clinical symptom of glaucoma. Gradual apoptosis of retinal ganglion cells (RGCs) due to elevated intraocular pressure, as well as retinal neovascularization, lead to thinning of retinal nerve fiber layer and visual field damage $(1,2)$. Clinical studies in recent years provided increasing number of evidences that suggest aberrant immune activity may play an important role in occurrence of glaucoma. The response of the immune system to an immune stimulus, such as pathological intraocular pressure elevation, can cause optic nerve damage directly or indirectly, suggesting that the immune system plays an important role in regulation of RGCs $(3,4)$. In the pathogenesis of glaucoma, vascular endothelial growth factor (VEGF) plays an important role in induction of neovascularization (5). In an animal study, it was reported that intravitreal injection of VEGF induced formation of new blood vessels, which disappeared following injection of anti-VEGF drug (6). Ranibizumab is an antiVEGF drug that shows good efficacy profile. Cytokines also play an important role in the pathogenesis of glaucoma. The cytokine interleukin-6 (IL-6) binds to the signal transducing transmembrane subunit gp130, thus activating the STAT3 pathway which regulates the expression of Bcl-xL and Bcl-2. Upregulation of anti-apoptotic $\mathrm{Bcl}-\mathrm{xL}$ and $\mathrm{Bcl}-2$ prevent apoptosis of $\operatorname{RGCs}(7,8)$. The purpose of this study was to investigate the effects of intravitreal injection of ranibizumab on the expression of IL-6 and VEGF in peripheral blood and aqueous humor of glaucoma rat model to evaluate the efficacy for treating optic nerve injury.

\section{Materials and methods}

Animal subjects. Specific Pathogen Free (SPF) SD rats were purchased from Wuhan Hualianke Biotechnology Co., Ltd. (Wuhan, China). The rats, aged 7-11 days and weighing 16-25 g, were fed with SPF ShooBree, rat food obtained from Jiangsu Province Collaborative Pharmaceutical Bioengineering Co., 
Table I. General information of rat subjects in both groups.

\begin{tabular}{lcccr}
\hline Items & Observation group $(\mathrm{n}=40)$ & Control group $(\mathrm{n}=40)$ & $\mathrm{t}$ & P-value \\
\hline Age (days) & $8.2 \pm 1.3$ & $9.8 \pm 1.6$ & 0.226 & 0.899 \\
Sex (male/female) & $24 / 16$ & $19 / 21$ & 0.967 & 0.645 \\
Weight $(\mathrm{g})$ & $22.4 \pm 3.8$ & $26.7 \pm 4.2$ & 0.742 & 0.785 \\
Intraocular pressure $(\mathrm{mm} / \mathrm{Hg})$ after anesthesia & $17.1 \pm 2.1$ & $16.7 \pm 1.8$ & 0.623 & 0.812 \\
Intraocular pressure $(\mathrm{mm} / \mathrm{Hg})$ on day 1 & $27.6 \pm 2.1$ & $28.4 \pm 1.5$ & 0.569 & 0.865 \\
Intraocular pressure $(\mathrm{mm} / \mathrm{Hg}$ ) on day 7 & $26.4 \pm 1.7$ & $28.1 \pm 2.3$ & 0.857 & 0.742 \\
Intraocular pressure $(\mathrm{mm} / \mathrm{Hg}$ ) on day 14 & $20.4 \pm 2.1$ & $31.1 \pm 2.8$ & 2.973 & 0.037 \\
Intraocular pressure $(\mathrm{mm} / \mathrm{Hg}$ ) on day 21 & $17.8 \pm 1.9$ & $31.4 \pm 3.1$ & 4.569 & 0.019 \\
\hline
\end{tabular}

Ltd. (Jiangsu, China). All rats were maintained in a controlled environment with a light/dark cycle of $12 \mathrm{~h}$, a temperature of $21 \pm 2^{\circ} \mathrm{C}$ and a humidity of $30-70 \%$, and were allowed free access to food and drinking water. The food tray and water bottle were replaced 1-2 times a week with fresh food and water. The study was approved by the Ethics Committee of People's Hospital of Dongying (Dongying, China).

SD rat glaucoma model establishment. One hundred and twenty-five SD rats were given intraperitoneal injection of pentobarbital sodium $(50 \mathrm{mg} / \mathrm{kg})$. After satisfactory induction of general anesthesia, topical ocular anesthesia was given by instilling oxybuprocaine hydrochloride eye drops (4 g/l) into the conjunctival sac. Then, the rat intraocular pressure was measured three times and the average was recorded. After the rat head was positioned under a microscope (Lufeifan Biotecs, Jiaozuo, China), laser photocoagulation was performed on the scleral vein by using a 532-nm diode laser at 0.075 -watt laser power and 0.3-sec duration. Approximately 150 laser burns were delivered in each eye. After the procedure, lincomycin hydrochloride and erythromycin were applied to reduce inflammatory reactions in the eye. On the second day (day 2), a slit-lamp microscopic examination was performed to evaluate corneal edema, conjunctival hyperemia and anterior chamber reaction. Intraocular pressure was measured three times on the day after operation (day 1), day 7, 14 and 21, and the average values were recorded. The rat glaucoma model establishment was successful if the increase of intraocular pressure was no $<15 \mathrm{mmHg}$ on day 7 .

After model establishment, the rats were randomly divided into two groups, the control group $(n=40)$ and the observation group $(n=40)$. Rats in the observation group were treated by intravitreal injections of ranibizumab (Shanghai TheraMabs Biotechnology Co., Ltd., Shanghai, China) at a dose of $0.05 \mathrm{ml} /$ day of $10 \mathrm{mg} / \mathrm{ml}$ solution. Drug treatment in this group continued until the end of the experiment. Rats in the control group received no treatment.

Indicators observed. Rats in the two groups were sacrificed by decapitation on day $7(n=13)$, day $14(n=13)$ and day 21 $(n=14)$ after start of treatment. Seven days before sacrifice, retrograde labeling of all RGCs was performed by bilateral injection of fluorogold $(0.5 \mu \mathrm{l}, 50 \mathrm{~g} / \mathrm{l})$ into superior colliculi. After rats were sacrificed at the time-points, the retina was harvested. Numbers of RGCs were counted under a fluo- rescence microscope (Lufeifan Biotecs). In the meantime, whole blood and aqueous humor were collected. The levels of IL-6 and VEGF in whole blood and aqueous humor were determined by using an enzyme-linked immunosorbent assay (ELISA) kit for IL-6 and an ELISA kit for VEGF, respectively (Shanghai Jingkang Biological Engineering Co., Ltd., Shanghai, China).

Statistical analysis. Statistical software SPSS 19.0 [AsiaAnalytics (formerly SPSS China), Shanghai, China] was used in data processing. Measurement data were expressed as mean $\pm \mathrm{SD}$. Chi-square test, non-parametric K-S test and t-test were used, respectively, for comparison of rate, comparison between two groups and comparison between different time-points within a group. Comparison between multiple groups was done using one-way ANOVA test followed by post hoc test (Least Significant Difference). Correlations of IL-6 and VEGF with RGCs were examined by using logistic regression analysis. A difference was statistically significant if $\mathrm{P}<0.05$.

\section{Results}

General information of animal subjects. Of 125 SD rats that were used for establishment of rat glaucoma model, 80 were successful, 15 died (accidental death caused by improper operation) and 30 failed (with the increase of intraocular pressure $<15 \mathrm{mmHg}$ ). The success rate was $64 \%$. Rats in the observation group ( 24 males and 16 females) were aged $8.2 \pm 1.3$ days and weighed $22.4 \pm 3.8 \mathrm{~g}$, and rats in the control group (19 males and 21 females) were aged $9.8 \pm 1.6$ days and weighed $26.7 \pm 4.2 \mathrm{~g}$. The rats in both groups were allowed free access to food and water. There were no statistically significant differences in sex, age and weight between the two groups $(\mathrm{P}>0.05)$. Compared with the intraocular pressure on day 1 , the decline started to be statistically significant when the ranibizumab treatment reached day $14(\mathrm{P}<0.05)$. The detailed results are shown in Table I.

IL-6 levels in peripheral blood and aqueous humor of rats in both groups. Levels of IL-6 in peripheral blood and aqueous humor were quantitatively analyzed by ELISA and the results are shown in Table II. In the observation group, the levels of IL-6 in peripheral blood and aqueous humor decreased gradually over the treatment time, and the level of IL- 6 on day 21 
Table II. Levels of IL-6 (pg/ml) in rat peripheral blood and aqueous humor.

\begin{tabular}{lccccccc}
\hline & \multicolumn{3}{c}{ Peripheral blood } & & \multicolumn{3}{c}{ Aqueous humor } \\
\cline { 2 - 3 } Groups & Day 7 $(\mathrm{n}=13)$ & Day 14 $(\mathrm{n}=13)$ & Day 21 $(\mathrm{n}=14)$ & & Day 7 (n=13) & Day 14 (n=13) & Day 21 (n=14) \\
\hline Observation group & $10.13 \pm 2.21$ & $9.03 \pm 1.54$ & $7.63 \pm 1.24^{\mathrm{a}}$ & & $9.45 \pm 1.38$ & $8.67 \pm 1.52$ & $5.78 \pm 1.11^{\mathrm{b}}$ \\
Control group & $13.31 \pm 1.94$ & $14.96 \pm 2.68$ & $15.44 \pm 2.49^{\mathrm{c}}$ & & $11.44 \pm 2.01$ & $12.19 \pm 3.11$ & $13.97 \pm 2.94^{\mathrm{d}}$ \\
t-test & 3.136 & 3.445 & 4.237 & & 3.001 & 3.711 & 3.952 \\
P-value & 0.031 & 0.028 & 0.021 & & 0.034 & 0.027 & 0.023 \\
\hline
\end{tabular}

${ }^{\mathrm{a}} \mathrm{P}=0.024$, compared with the IL- 6 level on day 7 in peripheral blood within the same group; ${ }^{\mathrm{b}} \mathrm{P}=0.023$, compared with the IL- 6 level on day 7 in aqueous humor within the same group; ${ }^{\mathrm{P}} \mathrm{P}=0.032$, compared with the IL-6 level on day 7 in peripheral blood within the same group; ${ }^{\mathrm{d}} \mathrm{P}=0.034$, compared with the IL-6 level on day 7 in aqueous humor within the same group. IL-6, interleukin-6.

Table III. Levels of VEGF (pg/ml) in rat peripheral blood and aqueous humor.

\begin{tabular}{lccccccc}
\hline & \multicolumn{3}{c}{ Peripheral blood } & & \multicolumn{3}{c}{ Aqueous humor } \\
\cline { 2 - 4 } Groups & Day 7 $(\mathrm{n}=13)$ & Day 14 $(\mathrm{n}=13)$ & Day 21 $(\mathrm{n}=14)$ & & Day 7 $(\mathrm{n}=13)$ & Day 14 $(\mathrm{n}=13)$ & Day 21 $(\mathrm{n}=14)$ \\
\hline Observation group & $43.57 \pm 6.47$ & $36.14 \pm 9.44$ & $31.32 \pm 5.33^{\mathrm{a}}$ & & $40.14 \pm 4.24$ & $34.59 \pm 8.33$ & $27.17 \pm 3.62^{\mathrm{b}}$ \\
Control group & $68.44 \pm 11.39$ & $67.45 \pm 3.44$ & $66.34 \pm 9.54$ & & $57.11 \pm 5.64$ & $54.64 \pm 7.58$ & $55.18 \pm 4.49$ \\
t-test & 3.115 & 4.237 & 4.913 & & 3.445 & 3.816 & 4.569 \\
P-value & 0.032 & 0.021 & 0.013 & & 0.028 & 0.024 & 0.019 \\
\hline
\end{tabular}

${ }^{\mathrm{a}} \mathrm{P}=0.023$, compared with the VEGF level on day 7 in peripheral blood within the same group; ${ }^{\mathrm{b}} \mathrm{P}=0.018$, compared with the VEGF level on day 7 in aqueous humor within the same group.

was significantly different from that on day $7(\mathrm{P}<0.05)$. In the control group, the levels of IL- 6 in peripheral blood and aqueous humor increased gradually over time, and the level of IL-6 on day 21 was significantly different from that on day 7 $(\mathrm{P}<0.05)$. At the same time-point, the levels of IL-6 in serum and aqueous humor were higher in the control group than those in the observation group $(\mathrm{P}<0.05)$.

VEGF levels in peripheral blood and aqueous humor of rats in both groups. Levels of VEGF in peripheral blood and aqueous humor were quantitatively analyzed by ELISA and the results are shown in Table III. In the observation group, the levels of VEGF in peripheral blood and aqueous humor decreased gradually over the treatment time, and the level of VEGF on day 21 was significantly different from that on day $7(\mathrm{P}<0.05)$. In the control group, the levels of VEGF in peripheral blood and aqueous humor did not show significant differences between different time-points $(P>0.05)$. At the same time-point, the levels of VEGF in serum and aqueous humor were higher in the control group than those in the observation group $(\mathrm{P}<0.05)$.

Rat RGC counts in both groups. Rat RGCs were counted after fluorogold labeling. The count results were shown in Table IV. In the observation group, the number of RGCs increased gradually over the treatment time. The number difference between day 7 and 14, as well as between day 7 and 21, was statistically significant $(\mathrm{P}<0.05)$. In the control group, the number of RGCs decreased gradually over time. The differences between
Table IV. RGC counts in both groups.

\begin{tabular}{lccc}
\hline & \multicolumn{3}{c}{ RGC counts } \\
\cline { 2 - 4 } Groups & Day 7 (n=13) & Day 14 $(\mathrm{n}=13)$ & Day 21 (n=14) \\
\hline Observation & $185.41 \pm 28.44$ & $191.12 \pm 31.32^{\mathrm{a}}$ & $197.24 \pm 16.51^{\mathrm{b}}$ \\
group & & & \\
Control group & $145.25 \pm 15.86$ & $128.94 \pm 18.54^{\mathrm{c}}$ & $104.39 \pm 13.24^{\mathrm{d}}$ \\
t-test & 3.012 & 3.255 & 4.673 \\
P-value & 0.033 & 0.027 & 0.016
\end{tabular}

${ }^{\mathrm{a}} \mathrm{P}=0.036$, compared with the number of RGCs on day 7 ; ${ }^{\mathrm{b}} \mathrm{P}=0.028$, compared with the number of RGCs on day 7 ; ${ }^{\mathrm{c}} \mathrm{P}=0.031$, compared with the number of RGCs on day $7 ;{ }^{d} \mathrm{P}=0.025$, compared with the RGCs number on day 7 , and $\mathrm{P}=0.030$, compared with the RGCs number on day 14. RGCs, retinal ganglion cells.

every two time-points were significant $(\mathrm{P}<0.05)$. At the same time-point, the number of RGCs was lower in the control group than that in the observation group $(\mathrm{P}<0.05)$.

Correlations of IL-6 and VEGF with RGC counts. Correlations of IL- 6 and VEGF with RGC counts were evaluated by logistic regression analysis. The analysis revealed that IL-6 and RGC counts, as well as VEGF and RGC counts, were negatively correlated. As the number of RGCs increased, the levels of IL-6 and VEGF gradually decreased $(r=-0.743$ and $P=0.012$ for IL-6; r=-0.675 and P=0.022 for VEGF) (Fig. 1). 
A

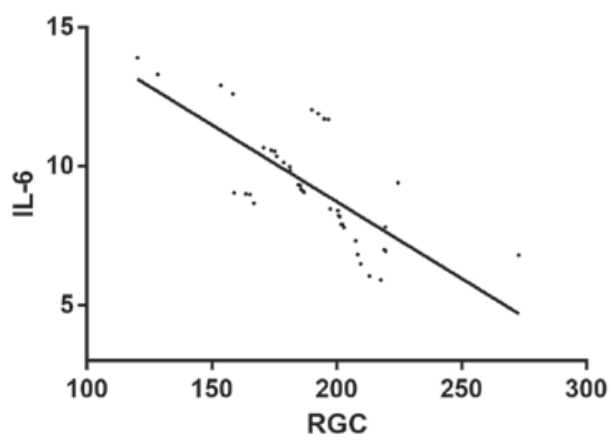

B

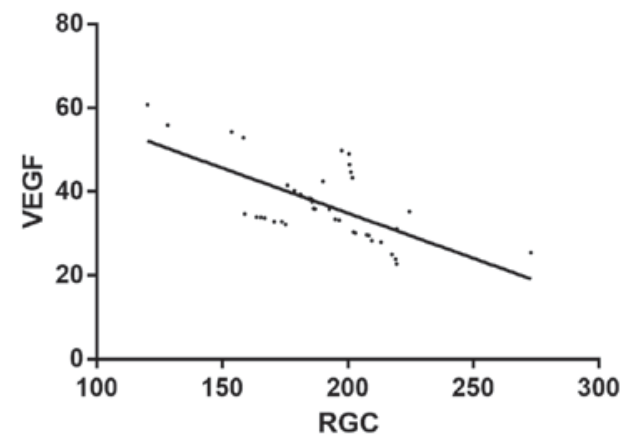

Figure 1. Logisitic regression analysis of the association of IL-6 and VEGF and RGCs. RGCs, retinal ganglion cells; IL-6, interleukin-6; VEGF, vascular endothelial growth factor.

\section{Discussion}

Glaucoma leading to optic nerve injury and visual loss is a non-negligible public health issue globally. According to reports, the number of patients with glaucoma could reach up to 80 million in the world, and 6 million in China alone by $2020(9,10)$. Glaucoma has a significant negative impact on vision function, however, it does not respond well to treatment of drugs and surgery. The primary goal of current treatments for glaucoma is focused on reduction of symptoms $(11,12)$. In this study, therapeutic efficacy of ranibizumab in treatment of visual impairment due to glaucoma was evaluated by monitoring levels of IL-6 and VEGF, and the correlations of IL-6 and VEGF with visual impairment were examined.

Successful establishment of glaucoma rat model was indicated by a difference of no less than $15 \mathrm{mmHg}$ in intraocular pressures before and after the model establishment. All rats in this study, as well as the rat food, were SPF grade. Experimental results showed that the number of RGCs labeled with fluorogold was higher in the observation group treated with ranibizumab than that in the control group $(\mathrm{P}<0.05)$. Ranibizumab is a recombinant humanized monoclonal antibody fragment. Clinically it was demonstrated to alleviate macular edema, and reduce neovascularization and vascular leakage in patients by effectively antagonizing VEGF $(13,14)$. In this study, the levels of VEGF in rat serum and aqueous humor were determined, and it was found that the VEGF levels of rats treated with ranibizumab were significantly lower $(\mathrm{P}<0.05)$ than those of the control group, and decreased further over time in the treatment. Improvement of VEGF levels by ranibizumab was also reported by Froger $e t$ al and Molokhia et al $(15,16)$. The levels of IL-6 were also measured by ELISA, which showed that the IL- 6 levels of rats treated with ranibizumab were significantly lower $(\mathrm{P}<0.05)$ than those of the control group. Similar to VEGF, the IL-6 levels decreased further over time in the treatment as well. Therefore, ranibizumab improved not only the VEGF levels but also the IL-6 levels, which was very important in alleviating the inflammatory response. Currently more studies of ranibizumab were focused on its effect on improving the VEGF levels $(17,18)$ and there has been no report in literature about its effect on improving the IL-6 levels. Thus, this study represents the first study of ranibizumab on its effect on improving the IL-6 levels. Due to using experimental animal model in this study, the findings need further support from studies by larger sample sets and clinical studies. Correlations of IL-6 and VEGF levels with optic nerve injury were also examined in this study. Using RGC count as an indicator of the extent of optic nerve injury, it was found that the RGC count was higher when levels of IL-6 and VEGF were lower, suggesting the levels of IL-6 and VEGF were negatively correlated with optic nerve damage. Thus, lower levels of IL-6 and VEGF indicate lower extent of optic nerve injury. Johnson et al reported that elevated IL-6 levels caused damage to the optic nerve head, compromising the axonal integrity (19). In Fisher J's study, microglia cell survival was increased in IL-6 gene knockout mice (20). Microglia cells play an important role in the sequelae of neurological injury. These reports confirmed that increased IL-6 levels resulted in optic nerve injury, which were in accordance with the findings in this study. Bennett et al reported that decreased VEGF levels improved papilledema, as well as visual impairment (21). This was consistent with our findings. The findings in this study were obtained by establishing and using animal models. Further studies using larger sample set and clinical studies are needed to support these findings.

In conclusion, ranibizumab alleviated optic nerve injury by reducing levels of IL- 6 and VEGF in peripheral blood and aqueous humor of glaucoma rat model.

\section{Acknowledgements}

Not applicable.

\section{Funding}

No funding was received.

\section{Availability of data and materials}

The datasets used and/or analyzed during the present study are available from the corresponding author on reasonable request.

\section{Authors' contributions}

YS, QS and LL conceived and designed the study. YS, JX and XL were responsible for the collection and analysis of 
the experimental data. QS and LL interpreted the data and drafted the manuscript. YS, QS and JX revised the manuscript critically for important intellectual content. All authors have read and approved the final manuscript.

\section{Ethics approval and consent to participate}

The study was approved by the Ethics Committee of People's Hospital of Dongying (Dongying, China).

\section{Patient consent for publication}

Not applicable.

\section{Competing interests}

The authors declare that they have no competing interests.

\section{References}

1. Tham YC, Li X, Wong TY, Quigley HA, Aung T and Cheng CY: Global prevalence of glaucoma and projections of glaucoma burden through 2040: A systematic review and meta-analysis. Ophthalmology 121: 2081-2090, 2014.

2. Weinreb RN, Aung T and Medeiros FA: The pathophysiology and treatment of glaucoma: A review. JAMA 311: 1901-1911, 2014.

3. Jia Y, Wei E, Wang X, Zhang X, Morrison JC, Parikh M, Lombardi LH, Gattey DM, Armour RL, Edmunds B, et al: Optical coherence tomography angiography of optic disc perfusion in glaucoma. Ophthalmology 121: 1322-1332, 2014.

4. Gramlich OW, Ding QJ, Zhu W, Cook A, Anderson MG and Kuehn MH: Adoptive transfer of immune cells from glaucomatous mice provokes retinal ganglion cell loss in recipients. Acta Neuropathol Commun 3: 56, 2015.

5. Sun Y, Liang Y, Zhou P, Wu H, Hou X, Ren Z, Li X and Zhao M: Anti-VEGF treatment is the key strategy for neovascular glaucoma management in the short term. BMC Ophthalmol 16: $150,2016$.

6. Gu X, Yu X and Dai H: Intravitreal injection of ranibizumab for treatment of age-related macular degeneration: Effects on serum VEGF concentration. Curr Eye Res 39: 518-521, 2014

7. Berg K, Hadzalic E, Gjertsen I, Forsaa V, Berger LH, Kinge B, Henschien H, Fossen K, Markovic S, Pedersen TR, et al: Ranibizumab or bevacizumab for neovascular age-related macular degeneration according to the lucentis compared to avastin study treat-and-extend protocol: Two-year results. Ophthalmology 123 : 51-59, 2016.

8. Andreoli MT, Pinnolis M, Kieser T, Sun J and Andreoli CM: Feasibility and efficacy of a mass switch from ranibizumab (Lucentis) to bevacizumab (Avastin) for treatment of neovascular age-related macular degeneration. Digit J Ophthalmol 21: 1-17, 2015.

9. Kapetanakis VV, Chan MPY, Foster PJ, Cook DG, Owen CG and Rudnicka AR: Global variations and time trends in the prevalence of primary open angle glaucoma (POAG): A systematic review and meta-analysis. Br J Ophthalmol 100: 86-93, 2016.
10. Liu L, Jia Y, Takusagawa HL, Pechauer AD, Edmunds B, Lombardi L, Davis E, Morrison JC and Huang D: Optical coherence tomography angiography of the peripapillary retina in glaucoma. JAMA Ophthalmol 133: 1045-1052, 2015.

11. Kyari F, Wormald R, Murthy GV, Evans JR and Gilbert CE; Nigeria National Blindness and Visual Impairment Study Group: Ethnicity and deprivation are associated with blindness among adults with primary glaucoma in nigeria: Results from the nigeria national blindness and visual impairment survey. J Glaucoma 25: e861-e872, 2016.

12. Gharahkhani P, Burdon KP, Fogarty R, Sharma S, Hewitt AW, Martin S, Law MH, Cremin K, Bailey JNC, Loomis SJ, et al; Wellcome Trust Case Control Consortium 2, NEIGHBORHOOD consortium: Common variants near ABCA1, AFAP1 and GMDS confer risk of primary open-angle glaucoma. Nat Genet 46: 1120-1125, 2014.

13. Narayanan R, Panchal B, Das T, Chhablani J, Jalali S, Ali MH; MARVEL study group: A randomised, double-masked, controlled study of the efficacy and safety of intravitreal bevacizumab versus ranibizumab in the treatment of macular oedema due to branch retinal vein occlusion: MARVEL Report No. $1 . \mathrm{Br}$ J Ophthalmol 99: 954-959, 2015.

14. Türkcü FM, Cinar Y, Türkcü G, Sahin A, Cingü AK, Yüksel H, Sahin M, Yıldırım A and Caça I: Topical and subconjunctival ranibizumab (lucentis) for corneal neovascularization in experimental rat model. Cutan Ocul Toxicol 33: 138-144, 2014.

15. Froger NG, Forster V, Ivkovic I, Matonti F, Sahel JA and Picaud SA: Ranibizumab (Lucentis ${ }^{\circledR}$ ) suppresses the autocrine VEGF-elicited survival of purified retinal ganglion cells. Invest Ophthalmol Vis Sci 55: 2391-2391, 2014.

16. Molokhia S, Burr RM, Flood M, Vallrath M, Winter G and Ambati BK: Lens capsule biodegradable lipid implant for sustained-release anti-VEGF therapy of neovascular AMD. Invest Ophthalmol Vis Sci 57: 4004-4004, 2016.

17. Korhonen T: Developing Medical Record for Follow-Up of Wet Age-Related Macular Degeneration. Leadership, Innovation and Entrepreneurship as Driving Forces of the Global Economy. Springer International Publishing, pp77-83, 2017.

18. Niwa $Y$, Kakinoki $M$, Sawada T, Wang $X$ and Ohji $M$ : Ranibizumab and aflibercept: Intraocular pharmacokinetics and their effects on aqueous VEGF level in vitrectomized and nonvitrectomized macaque eyes. Invest Ophthalmol Vis Sci 56: 6501-6505, 2015.

19. Johnson EC, Doser TA, Cepurna WO, Dyck JA, Jia L, Guo Y, Lambert WS and Morrison JC: Cell proliferation and interleukin-6-type cytokine signaling are implicated by gene expression responses in early optic nerve head injury in rat glaucoma. Invest Ophthalmol Vis Sci 52: 504-518, 2011.

20. Fisher J, Mizrahi T, Schori H, Yoles E, Levkovitch-Verbin H, Haggiag S, Revel M and Schwartz M: Increased post-traumatic survival of neurons in IL-6-knockout mice on a background of EAE susceptibility. J Neuroimmunol 119: 1-9, 2001.

21. Bennett JL, Thomas S, Olson JL and Mandava N: Treatment of nonarteritic anterior ischemic optic neuropathy with intravitreal bevacizumab. J Neuroophthalmol 27: 238-240, 2007.

This work is licensed under a Creative Commons Attribution-NonCommercial-NoDerivatives 4.0 International (CC BY-NC-ND 4.0) License. 